\title{
Desynchronization of Auditory Steady-State Responses Related to Changes in Interaural Phase Difference: an Objective Measure of Binaural Hearing
}

Charlotte Vercammen ${ }^{\mathrm{a}}$, Astrid van Wieringen ${ }^{\mathrm{a}}$, Jan Wouters ${ }^{\mathrm{a}}$ and Tom Francart ${ }^{\mathrm{a}}$

${ }^{a} \mathrm{KU}$ Leuven - University of Leuven, Department of Neurosciences, Research Group Experimental Oto-rhino-laryngology, Onderwijs en Navorsing 2, Herestraat 49 bus 721, 3000 Leuven, Belgium.

Corresponding author: Charlotte Vercammen, KU Leuven - University of Leuven, Department of Neurosciences, ExpORL, Onderwijs en Navorsing 2, Herestraat 49 bus 721, 3000 Leuven, Belgium. E-mail: Charlotte.Vercammen@kuleuven.be

$\begin{array}{llll}\text { ASSRs } & \text { Auditory Steady-State Responses } & \mathrm{f}_{\mathrm{c}} & \text { carrier frequency } \\ \text { BIC } & \text { Binaural Interaction Component } & \mathrm{f}_{\mathrm{m}} & \text { modulation frequency } \\ \text { BMLD } & \text { Binaural Masking Level Difference } & \text { ILD } & \text { interaural level difference } \\ \text { CAEPs } & \text { Cortical Auditory Evoked Potentials } & \text { IPD } & \text { interaural phase difference } \\ \text { CMS } & \text { Common Mode Sense } & \text { ITD } & \text { interaural time difference } \\ \text { DRL } & \text { Driven Right Leg } & \text { MEG } & \text { magnetoencephalography } \\ \text { DSS } & \text { Denoising Source Separation } & \text { SAM } & \text { sinusoidal amplitude } \\ \text { EEG } & \text { electroencephalography } & & \text { modulated }\end{array}$

Keywords: electroencephalography, auditory steady-state responses, binaural hearing, interaural phase differences, modulation frequency 


\title{
Desynchronization of Auditory Steady-State Responses Related to Changes in Interaural Phase Difference: an Objective Measure of Binaural Hearing
}

\begin{abstract}
Objective

Binaural processing can be measured objectively as a desynchronization of phase locked neural activity to changes in interaural phase differences (IPDs). This was reported in a magnetoencephalography study for $40 \mathrm{~Hz}$ amplitude modulated tones by Ross [Journal of Neurophysiology, 100, 1265-1277 (2008)]. The goal of this study was to measure this desynchronization using electroencephalography and explore the outcomes for different modulation frequencies.
\end{abstract}

Design

Auditory steady-state responses (ASSRs) were recorded to pure tones, amplitude modulated at 20,40 , or $80 \mathrm{~Hz}$. IPDs switched between $0^{\circ}$ and $180^{\circ}$ at fixed time intervals.

Study Sample

Sixteen young listeners with bilateral normal hearing thresholds $(\leq 25 \mathrm{~dB} H \mathrm{HL}$ at .0125 $8 \mathrm{kHz}$ ) participated in this study.

\section{Results}

Significant ASSR phase desynchronizations to IPD changes were detected in 14 out of 16 participants for $40 \mathrm{~Hz}$ and in 8 , respectively 9, out of 13 participants for 20 and $80 \mathrm{~Hz}$ modulators. Desynchronization and restoration of ASSR phase took place significantly faster for $80 \mathrm{~Hz}$ than for 40 and $20 \mathrm{~Hz}$. 


\section{Conclusions}

ASSR desynchronization to IPD changes was successfully recorded using electroencephalography. It was feasible for 20,40 , and $80 \mathrm{~Hz}$ modulators and could be an objective tool to assess processing of changes in binaural information.

Keywords: electroencephalography, auditory steady-state responses, binaural hearing, interaural phase differences, modulation frequency 


\section{Introduction}

Normal hearing listeners can localize sound sources in the horizontal plane by processing interaural phase (IPDs) and level differences (ILDs) between sound waves arriving at the left and right ear. Such processing facilitates stream segregation and speech understanding in noise, for instance when attending to one speaker in the presence of others. In the human auditory system, binaural information is derived at brainstem level. When the ventral cochlear nuclei project to the lateral and/or medial superior olives, information is exchanged between the left and right auditory pathways. Thereafter, binaural information is projected to the midbrain and further up to the thalamus and auditory cortex (e.g. Tollin \& Yin, 2005; Grothe et al, 2010). Behavioral IPD and ILD detection thresholds as small as $10-20 \mu \mathrm{sec}$ (for $1000 \mathrm{~Hz}$ pure tones) and $2 \mathrm{~dB}$ (for 200-5000 Hz pure tones) have been reported, respectively (Klumpp \& Eady, 1956; Zwislocki \& Feldman, 1956; Yost \& Dye, 1988).

As the superior olives are the first stage of the central auditory system at which binaural information is derived (e.g. Tollin \& Yin, 2005; Grothe et al, 2010), it is expected that electrophysiological measures of brainstem activity would be able to objectively reflect binaural functioning. Both the binaural interaction component or BIC (Dobie \& Berlin, 1979; Dobie \& Norton, 1980; McPherson \& Starr, 1993) and electrophysiological measures of the binaural masking level difference or BMLD (Kevanishvili \& Lagidze, 1987; Galambos \& Makeig, 1992; Wong \& Stapells, 2004; Wilson \& Krishnan, 2005) have been proposed to do so. Yet, the clinical applications (Debruyne, 1984; Haywood et al, 2015) and underlying neural mechanisms of the BIC (Zhou \& Durrant, 2003; Dajani \& Picton, 2006) have been questioned. Also, BMLD results reported in literature are limited for brainstem activity (Wilson \& Krishnan, 2005; Clinard et al, 2016).

In 2008, Ross described an objective measure of binaural information processing using magnetoencephalography (MEG) for a $40 \mathrm{~Hz}$ modulator. By bilaterally presenting sinusoidally 
amplitude modulated tones (SAM), steady-state neural activity to the modulation frequency was induced. At fixed time intervals, changes in interaural phase of the carrier wave resulted in a transient desynchronization of ASSRs, reflected by a decrement in ASSR amplitude, a deviation in ASSR phase and the presence of cortical auditory evoked potentials (CAEPs) (Ross et al, 2007b; Ross, 2008). Ross (2008) reported phase desynchronizations as the most robust measure of processing changes in IPDs, as they were detected in more individuals than decrements in ASSR amplitude. Averaged across 12 young participants, he reported that ASSR phase deviations reached their maximum $150 \mathrm{~ms}$ after the IPDs switched between $0^{\circ}$ and $180^{\circ}$ for a $500 \mathrm{~Hz}$ carrier and a $40 \mathrm{~Hz}$ modulator. Approximately $350 \mathrm{~ms}$ later, phase locking to the modulation frequency was completely restored.

As electroencephalography (EEG) provides lower hardware costs than MEG, is a silent technique and does not result in magnetic interference with cochlear implants, an objective measure of IPD change processing using EEG would be advantageous for clinical practice. Based on the fine temporal resolution of EEG, we hypothesized that it would be feasible to accurately detect ASSR phase desynchronizations in reaction to IPD changes as a function of time. Furthermore, as the main neural sources of ASSRs are suggested to depend on modulation frequency (Giraud et al, 2000), it could provide insight in the underlying neural mechanisms to determine whether ASSRs elicited by different modulation frequencies would desynchronize in response to changes in binaural information. Ross (2008) used a $40 \mathrm{~Hz}$ modulation frequency to elicit ASSRs, which are expected to be generated by both cortical and subcortical neural sources (Giraud et al, 2000; Herdman et al, 2002). We hypothesized that if the phase desynchronization patterns would show morphological differences when applying different modulation frequencies, this would yield evidence that the underlying neural mechanisms are indeed different. We hypothesized that the point in time at which the phase deviation reached its maximum in response to IPD changes would be detected earlier $(<150 \mathrm{~ms})$ for brainstem 
sources and later (>150 ms) for cortico-thalamic sources in comparison to cortical and subcortical sources. If there would be no effect of modulation frequency on the point in time at which the phase deviation reached its maximum, similar neural generators would be involved for different modulation conditions and/or the method would not be sensitive enough to detect these differences. CAEPs, characterized by a $\mathrm{P}_{1} \mathrm{~N}_{1} \mathrm{P}_{2}$ change complex, were hypothesized to be detected with latencies similar to the ones reported by Tremblay et al (2007) and by Ross et al (2007b) for different modulation frequencies. In contrast to ASSRs, the main location at which CAEPs are generated, were expected to be independent of modulation frequency.

The first goal of the current study was to investigate whether ASSR phase desynchronizations could be measured using EEG. The second goal of this study was to explore the outcomes for different modulation frequencies. In addition to the $40 \mathrm{~Hz}$ condition, which was expected to elicit ASSRs generated by both cortical and subcortical neural sources (Giraud et al, 2000; Herdman et al, 2002), potential desynchronization was examined using stimuli that were amplitude modulated at $20 \mathrm{~Hz}$ or $80 \mathrm{~Hz}$. These modulation frequencies were expected to generate ASSRs from mainly cortico-thalamic $(20 \mathrm{~Hz})$ and mainly brainstem sources $(80 \mathrm{~Hz})$, respectively (Giraud et al, 2000; Herdman et al, 2002).

\section{Methods}

\section{Participants}

Sixteen young normal hearing participants took part in this study: nine women and seven men with a mean age of 22 years (age range: 18-28 years). All had normal hearing thresholds ( $\leq 25 \mathrm{~dB} \mathrm{HL}$ ) at octave frequencies from 0.125 to $8 \mathrm{kHz}$ in both ears. Pure-tone thresholds were determined by the Hughson-Westlake 5 up 10 down method (Carhart \& Jerger, 1959) in a double walled, soundproof booth by means of a clinical audiometer (Madsen Electronics) and TDH-39 earphones. 
The study was approved by the Medical Ethical Committee of the University Hospitals KU Leuven (approval number B322201214866) and all participants gave written informed consent.

\section{Set-up}

\section{Stimulation}

Stimuli, similar to those used by Ross (2008), were constructed in Matlab R2013a (The MathWorks Inc., 2013) by generating 100\% SAM tones with a carrier frequency of $500 \mathrm{~Hz}$ and modulation frequencies of 20,40 , or $80 \mathrm{~Hz}$ (referred to as the 20,40 , or $80 \mathrm{~Hz}$ condition). To ensure that each stimulus contained an integer number of cycles, carrier frequencies of 500, 520, and $480 \mathrm{~Hz}$ were used for the 20,40 , and $80 \mathrm{~Hz}$ modulation conditions, respectively (see Figure 1 for a schematic overview).
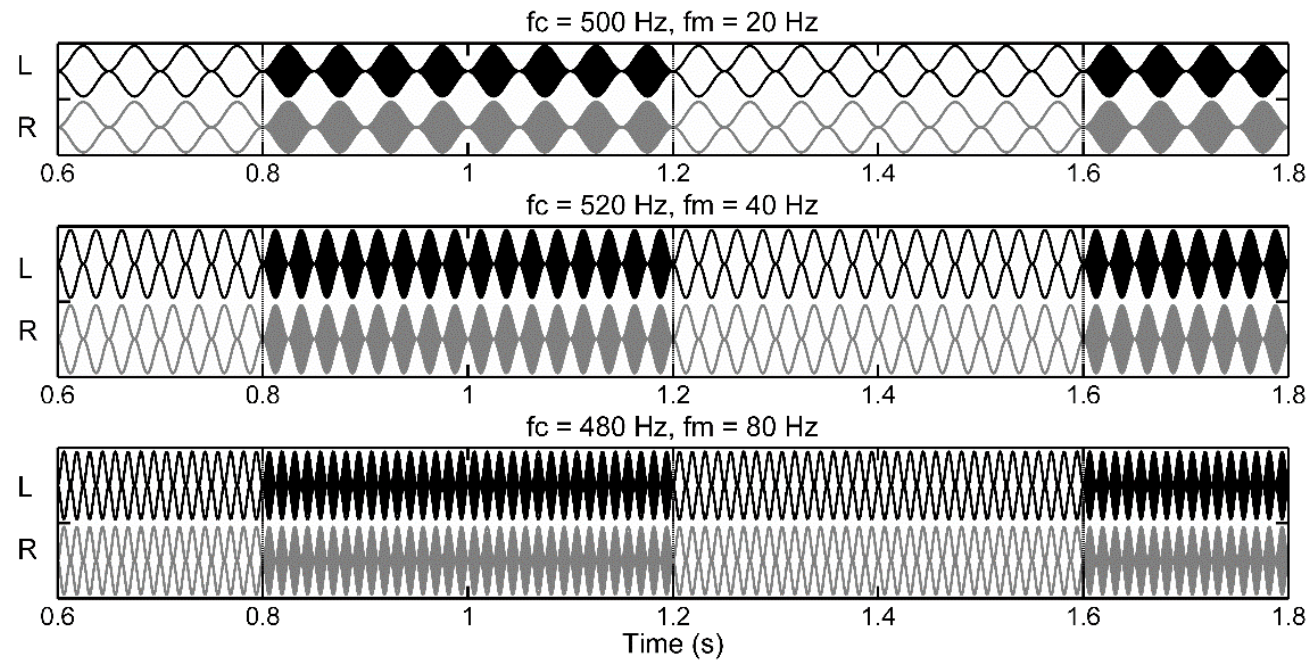

Figure 1. An overview of the three stimuli. From top to bottom: carrier waves of 500, 520, and $480 \mathrm{~Hz}, 100 \%$ amplitude modulated at a rate of 20,40 , and $80 \mathrm{~Hz}$, respectively. Black signals were presented to the left ear (also indicated by "L") and grey signals to the right ear (also indicated by " $R$ "). The total duration of the stimuli was $4 \mathrm{~s}$, of which a fragment is schematically illustrated here (time frame between $0.6-1.8 \mathrm{~s}$ ). Vertically dashed lines represent IPD changes in the carrier: the first is introduced at $0.8 \mathrm{~s}$, after which they recurred every $0.4 \mathrm{~s}$. Unfilled areas represent diotic listening situations (an IPD of $0^{\circ}$ between both ears). Filled areas represent dichotic listening situations (an IPD of $180^{\circ}$ between both ears). Alternately, the IPD changes resulted in transitions from diotic to dichotic listening situations (e.g. at 0.8 and $1.6 \mathrm{~s}$ ) and from dichotic to diotic listening situations (e.g. at $1.2 \mathrm{~s}$ ). All IPD changes were introduced when the modulation cycle reached a minimum, to prevent the acoustic change from causing distortions. 
Individual stimuli were four seconds long. During the first $0.8 \mathrm{~s}$, stimulation was diotic. At $0.8 \mathrm{~s}$, the phase of the carrier wave shifted $+90^{\circ}$ in the left ear and $-90^{\circ}$ in the right ear, leading to an IPD change of $180^{\circ}$. Phase shifts recurred every $0.4 \mathrm{~s}$, resulting in eight IPD changes per stimulus. The first, third, fifth and seventh IPD changes introduced a transition from a diotic (IPD of $0^{\circ}$ ) to a dichotic listening situation (IPD of $180^{\circ}$ ), while the second, fourth, sixth and eighth IPD changes introduced a transition from a dichotic (IPD of $180^{\circ}$ ) to a diotic listening situation (IPD of $0^{\circ}$ ).

Stimulation was carried out through custom written software (Hofmann \& Wouters, 2010), a laptop and a Hammerfall DSP Multiface II soundcard. After calibration by means of a Brüel and Kjær sound level meter (type 2250), ZC-0032 preamplifier and 2 cc coupler (type 4152), stimuli were presented bilaterally at an intensity of $65 \mathrm{~dB}$ SPL through ER-3A insert phones in blocks of $600 \mathrm{~s}$. Subsequent stimuli were separated by one second of silence. Two blocks were recorded for the $40 \mathrm{~Hz}$ condition and up to four blocks for the 20 and $80 \mathrm{~Hz}$ conditions to ensure sufficient measurement time for the additional modulation conditions. Total measurement time was kept below two hours and a short break was introduced every half hour. Note that three participants were part of a pilot study, during which the $40 \mathrm{~Hz}$ condition was applied, but not the 20 and $80 \mathrm{~Hz}$ conditions.

\section{Recordings}

Participants listened passively to the stimuli during recordings, while sitting comfortably in a reclining chair and watching a silent movie with subtitles. Recordings were made in a double-walled, soundproof and electromagnetically shielded booth by means of the BioSemi ActiveTwo system (Biosemi, 2010) for multichannel EEG recordings. Sixty-four active, $\mathrm{Ag}-\mathrm{AgCl}$ pin-type electrodes were mounted in head caps and positioned on the participant's head, based on the International 10/10 System (American Clinical 
Neurophysiology Society, 2006). The electrode set-up was complemented by a Common Mode Sense (CMS) active and a Driven Right Leg (DRL) passive electrode as common electrode and current return path, respectively. Care was taken to keep electrode offsets stable and below 30 $\mathrm{mV}$. The EEG signal was AD-converted and amplified by an ActiveTwo amplifier (sampling rate $=8192 \mathrm{~Hz}$; gain $=32.25 \mathrm{nV} / \mathrm{bit})$ and recorded by ActiView software (Biosemi, 2010).

\section{Data Processing}

Data processing was done in Matlab R2013a (The MathWorks Inc., 2013). The EEG signal was segmented into epochs of $4 \mathrm{~s}$ and high-pass filtered with a cut-off frequency of $2 \mathrm{~Hz}$ to remove the DC component of the signal. A Morlet wavelet filter with a wavelet center frequency of the modulation frequency $\left(f_{m}\right)$ was applied $\left(\frac{2.5}{f_{m}}\right.$ half-intensity width) to obtain the response amplitude and phase at the modulation frequency. The $5 \%$ epochs with largest peakto-peak amplitudes were rejected to eliminate artifacts. Thereafter, Denoising Source Separation or DSS (de Cheveigné \& Simon, 2008) was applied. DSS is an algorithm based on principal component analysis that enhances the signal-to-noise ratio and reduces dimensionality by designing a spatial filter to partition recorded activity into stimulus-related and stimulusunrelated components, based on a criterion of stimulus-evoked reproducibility. The first resulting component is proposed by de Cheveigné and Simon (2008) as a suitable candidate to summarize EEG data as a function of time. The analyses in this study are reported based on the first component, time domain averaged over epochs (4 $\mathrm{s}$ in duration) and over within-stimulus IPD changes. Per modulation frequency, data of two conditions will be discussed: one reflecting IPD changes that resulted in transitions from a diotic (IPD of $0^{\circ}$ ) to a dichotic listening situation (IPD of $180^{\circ}$ ) and one reflecting IPD changes that resulted in transitions from dichotic (IPD of $180^{\circ}$ ) to diotic listening situations (IPD of $0^{\circ}$ ). 


\section{Statistical Analyses}

Statistical analyses were performed in Matlab R2013a (The MathWorks Inc., 2013) and SPSS Statistics 23 (IBM Corp., 2013).

Detection of ASSRs and ASSR desynchronization. ASSR amplitude and phase were obtained from the complex value after filtering the signal with a Morlet wavelet, centered at the modulation frequency. Analyses were done at an individual level and mean response amplitude and phase were the result of vector averaging.

Steady-state neural activity at the modulation frequency was detected per participant and per modulation condition using the Rayleigh test (Rayleigh, 1880; Dobie \& Wilson, 1994; Cebulla et al, 2006). $P$ values were Bonferroni-Holm (Aickin \& Gensler, 1996; Nakagawa, 2004) corrected $(\alpha=.05)$. The Rayleigh test compared the observed distribution of phases to a random distribution of phases across the full stimulus duration of 4 s. A significant result indicated that the observed phase distribution was significantly different from a random distribution, which means that the neural responses were phase locked to the modulation frequency and thus, that ASSRs were detected. As ASSR phase synchronization is a prerequisite for determining ASSR phase desynchronization, a statistic based on phase information alone was chosen for ASSR detection.

For the participants and stimulus conditions, in which the Rayleigh test demonstrated phase synchronization, the Hotelling $T^{2}$ test (Hotelling, 1931) with Bonferroni-Holm (Aickin \& Gensler, 1996; Nakagawa, 2004) corrected $p$ values was applied $(\alpha=.05)$. By comparing the average ASSR - determined by amplitude and phase - during a test range to a baseline, in sliding time windows of $\frac{1}{f_{m}}$, the Hotelling $T^{2}$ test determined whether the ASSR desynchronized in response to changes in IPDs. The baseline was defined as the last $0.20 \mathrm{~s}$ before the IPD changes, for all three modulation conditions. The test range was defined as the time frame between 0.05 
and $0.25 \mathrm{~s}, 0.05$ and $0.20 \mathrm{~s}$ and 0.05 and $0.10 \mathrm{~s}$ after the IPD changes for the 20,40 , and $80 \mathrm{~Hz}$ conditions, respectively. A significant response reflected a change in ASSR phase and amplitude elicited by the IPD changes, i.e. binaural interaction. The Hotelling $T^{2}$ test was performed twice: once for IPD changes that resulted in transitions from a diotic (IPD of $0^{\circ}$ ) to a dichotic listening situation (IPD of $180^{\circ}$ ) and once for IPD changes that resulted in transitions from dichotic (IPD of $180^{\circ}$ ) to diotic listening situations (IPD of $0^{\circ}$ ).

Effect of IPD change and $f_{m}$ on phase deviation. In case of a significant ASSR desynchronization determined by the Hotelling $T^{2}$ test, the point in time (in seconds) at which the phase deviation reached its maximum after the IPD changes - further referred to as the peak phase latency - and the phase deviation amplitude (in degrees) were determined (see Figure 2). Within every modulation condition, Mann Whitney $U$ tests $(\alpha=.05)$ determined whether the peak phase latencies and phase deviation amplitudes were similar for IPD changes from $0^{\circ}$ to $180^{\circ}$ compared to the reversed transitions from $180^{\circ}$ to $0^{\circ}$. To assess whether modulation frequency had an effect on the amplitude and latency of the phase deviations, Kruskal-Wallis tests $(\alpha=.05)$ and post-hoc Mann Whitney $U$ tests with Bonferroni corrected levels of significance $\left(\alpha=\frac{.05}{3}=.017\right)$ were applied. Non parametric tests were chosen due to the limited sample sizes per modulation condition.

CAEP detection. For detection of CAEPs, the EEG signal was submitted to an additional low-pass filtering, with a cut-off frequency of $10 \mathrm{~Hz}$. Latencies (in seconds) were determined for $\mathrm{P}_{1}, \mathrm{~N}_{1}$ and $\mathrm{P}_{2}$ in the time domain, more specifically in the time frame between 0.05 and 0.25 $\mathrm{s}$ after the IPD changes. To assess whether modulation frequency had an effect on the latencies of $\mathrm{P}_{1}, \mathrm{~N}_{1}$ and $\mathrm{P}_{2}$, Kruskal-Wallis tests $(\alpha=.05)$ were applied. 


\section{Results}

\section{Detection of ASSRs and ASSR desynchronization}

The Rayleigh test with Bonferroni-Holm corrected $p$ values $(\alpha=.05)$ detected significant ASSRs for all participants and for all stimulus conditions $\left(f_{m}=20,40\right.$, or $\left.80 \mathrm{~Hz}\right)$. The Hotelling $T^{2}$ test with Bonferroni-Holm corrected $p$ values $(\alpha=.05)$ revealed a significant ASSR desynchronization in $14 / 16$ participants for the $40 \mathrm{~Hz}$ condition and in 10/13 participants for the 20 and $80 \mathrm{~Hz}$ conditions. This applied to IPD changes that resulted in transitions from a diotic (IPD of $0^{\circ}$ ) to dichotic stimulation (IPD of $180^{\circ}$ ). For IPD changes that resulted in transitions from a dichotic (IPD of $180^{\circ}$ ) to diotic stimulation (IPD of $0^{\circ}$ ), the Hotelling $T^{2}$ test with Bonferroni-Holm corrected $p$ values $(\alpha=$.05) detected significant ASSR desynchronizations in 10/16 participants for $40 \mathrm{~Hz}$, in $4 / 13$ participants for $20 \mathrm{~Hz}$ and in $6 / 13$ participants for $80 \mathrm{~Hz}$, respectively.

The Hotelling $T^{2}$ test used a combined approach based on both ASSR phase and amplitude. Further analyses focused on ASSR phase alone. Per participant and per modulation condition, the point in time in seconds at which the phase deviation reached its maximum (peak phase latency) and the corresponding size of the phase deviation in degrees (phase deviation amplitude) were determined. See Figure 2, for typical examples. Both for IPD changes from diotic (IPD of $0^{\circ}$ ) to dichotic stimulation (IPD of $180^{\circ}$ ) and for the reversed transitions, three data points were excluded from further analyses, as the phase deviation amplitudes were $\leq 0^{\circ}$, despite significant Hotelling $T^{2}$ outcomes. This resulted in samples of 8, 14, and 9 participants for the $20 \mathrm{~Hz}, 40 \mathrm{~Hz}$, and $80 \mathrm{~Hz}$ conditions, respectively, for IPD changes from diotic (IPD of $0^{\circ}$ ) to dichotic stimulation (IPD of $180^{\circ}$ ). For the reversed transition, the resulting samples consisted of 3, 9 and 5 participants for the $20 \mathrm{~Hz}, 40 \mathrm{~Hz}$, and $80 \mathrm{~Hz}$ conditions, respectively. 

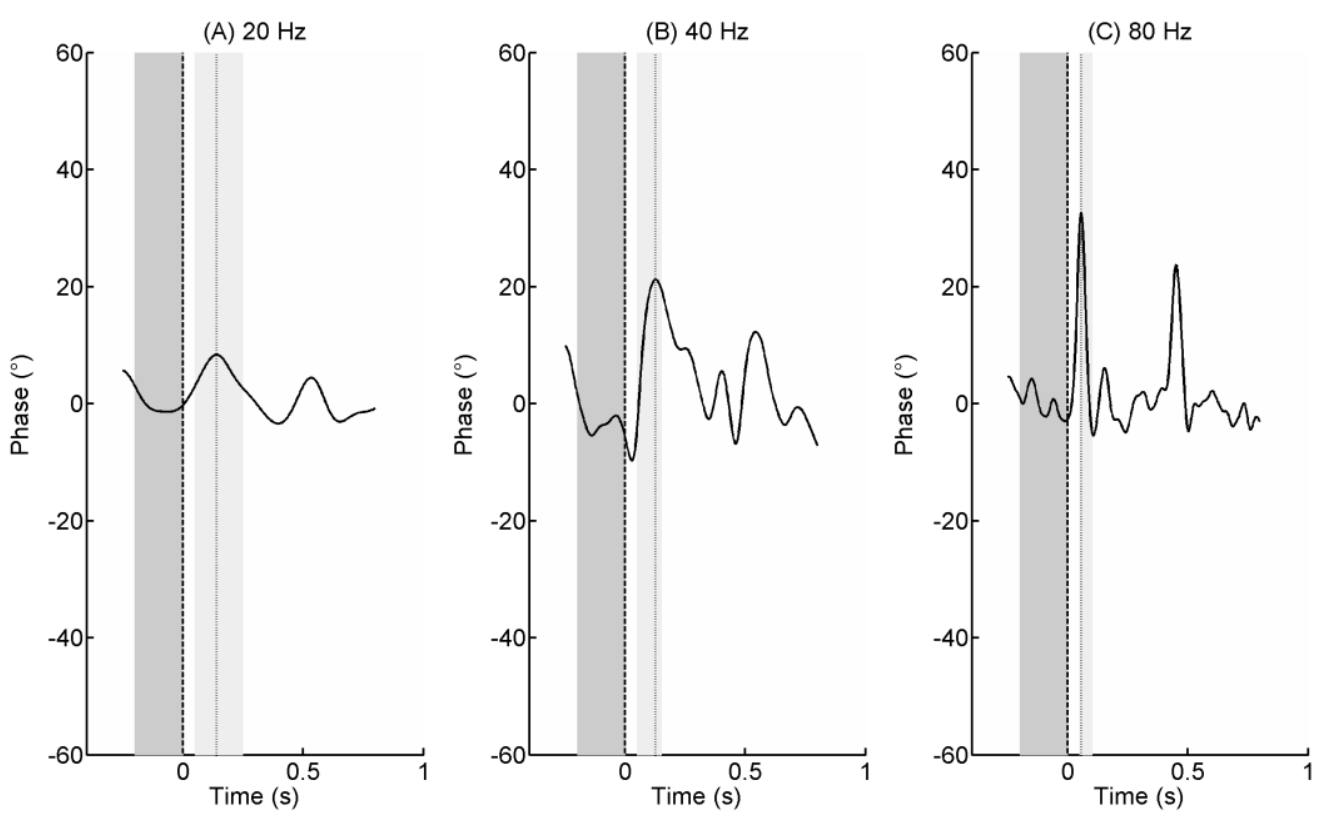

Figure 2. Typical examples of desynchronization and restoration of phase locked neural activity in response to IPD changes in the carrier wave, modulated at (A) $20 \mathrm{~Hz}$, (B) $40 \mathrm{~Hz}$ and (C) 80 $\mathrm{Hz}$. The first dashed line (referenced to $0 \mathrm{~s}$ ) indicates the point in time at which the IPD changed from $0^{\circ}$ to $180^{\circ}$. The average ASSR, determined in the time frame represented by the light grey coloring, is compared to a baseline represented by the dark grey coloring. The second dashed line represents the point in time at which the phase deviation reaches its maximum and at which the peak phase latency and phase deviation amplitude are determined.

\section{Effect of IPD change and $f_{m}$ on phase deviation}

Mann Whitney $U$ tests $(\alpha=.05)$ showed no significant difference between peak phase latencies for IPD changes from $0^{\circ}$ to $180^{\circ}$ compared to the reversed changes from $180^{\circ}$ to $0^{\circ}$. This was true for the 20,40 , and $80 \mathrm{~Hz}$ conditions $(20 \mathrm{~Hz}: U=3.50 ; z=-1.74 ; p=.08 ; r=.52$; $40 \mathrm{~Hz}: U=50.50 ; z=-.79 ; p=.43 ; r=.16 ; 80 \mathrm{~Hz}: U=22.00 ; z=-.07 ; p=.95 ; r=.02)$. Also, no significant differences in phase deviation amplitudes were detected for the three modulation conditions when comparing IPD changes from $0^{\circ}$ to $180^{\circ}$ to the reversed IPD changes $(20 \mathrm{~Hz}$ : $U=9.00 ; z=-.61 ; p=.54 ; r=0.18 ; 40 \mathrm{~Hz}: U=43.00 ; z=-1.26 ; p=.21 ; r=.26 ; 80 \mathrm{~Hz}: U=$ $11.00 ; z=-1.53 ; p=.13 ; r=.41)$. 
As the Hotelling $T^{2}$ test $(p<.01)$ detected significant ASSR desynchronizations in more participants for IPD changes from $0^{\circ}$ to $180^{\circ}$ than for IPD changes from $180^{\circ}$ to $0^{\circ}$, the former transitions were investigated further to determine whether modulation frequency influenced the peak phase latencies and phase deviation amplitudes.

A Kruskal-Wallis test $(\alpha=.05)$ with modulation frequency $(20,40$, and $80 \mathrm{~Hz})$ as independent variable and the peak phase latency (in seconds) as dependent variable showed that modulation frequency significantly affected the position at which the peak phase deviation was detected in response to the IPD changes $(H(2)=16.18 ; p<.001)$. Post-hoc Mann Whitney $U$ tests with Bonferroni corrected levels of significance $\left(\alpha=\frac{.05}{3}=.017\right)$ indicated that the peak phase latencies were significantly longer for the 20 and $40 \mathrm{~Hz}$ conditions when compared to those of the $80 \mathrm{~Hz}$ condition $(20-80 \mathrm{~Hz}: U=5.50 ; z=-2.94 ; p<.01 ; r=.71 ; 40-80 \mathrm{~Hz}: U=$ 9.00; $z=-3.41 ; p<.01 ; r=.71)$. Peak phase latencies for the 20 and $40 \mathrm{~Hz}$ conditions were not significantly different from each other $(U=22.50 ; z=-2.29 ; p=.02 ; r=.49)$.

A Kruskal-Wallis test $(\alpha=.05)$ with modulation frequency $(20,40$, and $80 \mathrm{~Hz})$ as independent variable and the phase deviation amplitude (in degrees) as dependent variable showed that modulation frequency also significantly affected the size of the phase deviations $(H(2)=10.30 ; p<.01)$. Post-hoc Mann Whitney $U$ tests with Bonferroni corrected levels of significance $\left(\alpha=\frac{.05}{3}=.017\right)$ showed that phase deviation amplitudes were significantly smaller for the $20 \mathrm{~Hz}$ compared to the $80 \mathrm{~Hz}$ condition $(20-80 \mathrm{~Hz}: U=7.00 ; z=-2.79 ; p<.01 ; r=.68)$. There was no significant difference in phase deviation amplitudes between the 20 and $40 \mathrm{~Hz}$ conditions and between the 40 and $80 \mathrm{~Hz}$ conditions $(20-40 \mathrm{~Hz}: U=29.00 ; z=-1.84 ; p=.07$; $r=.39 ; 40-80 \mathrm{~Hz}: U=28.00 ; z=-2.21 ; p=.03 ; r=.46)$.

For IPD changes from diotic (IPD of $0^{\circ}$ ) to dichotic stimulation (IPD of $180^{\circ}$ ), one data point was identified as an outlier for peak phase latency and three data points for phase deviation 
amplitude. This was based on an adapted version of the Tukey's resistant outlier labeling rule for non-normally distributed data with a g-value of 2.2 (Kimber, 1990; Iglewicz \& Banerjee, 2001). The abovementioned results were not influenced by excluding the data points identified as outliers from the sample, except for one comparison: a significant difference in phase deviation amplitudes was detected between the 40 and $80 \mathrm{H}$ conditions (40-80 Hz: $U=1.00 ; z$ $=-3.63 ; p<.001 ; r=.81)$. Figure 3 provides an overview of the results.
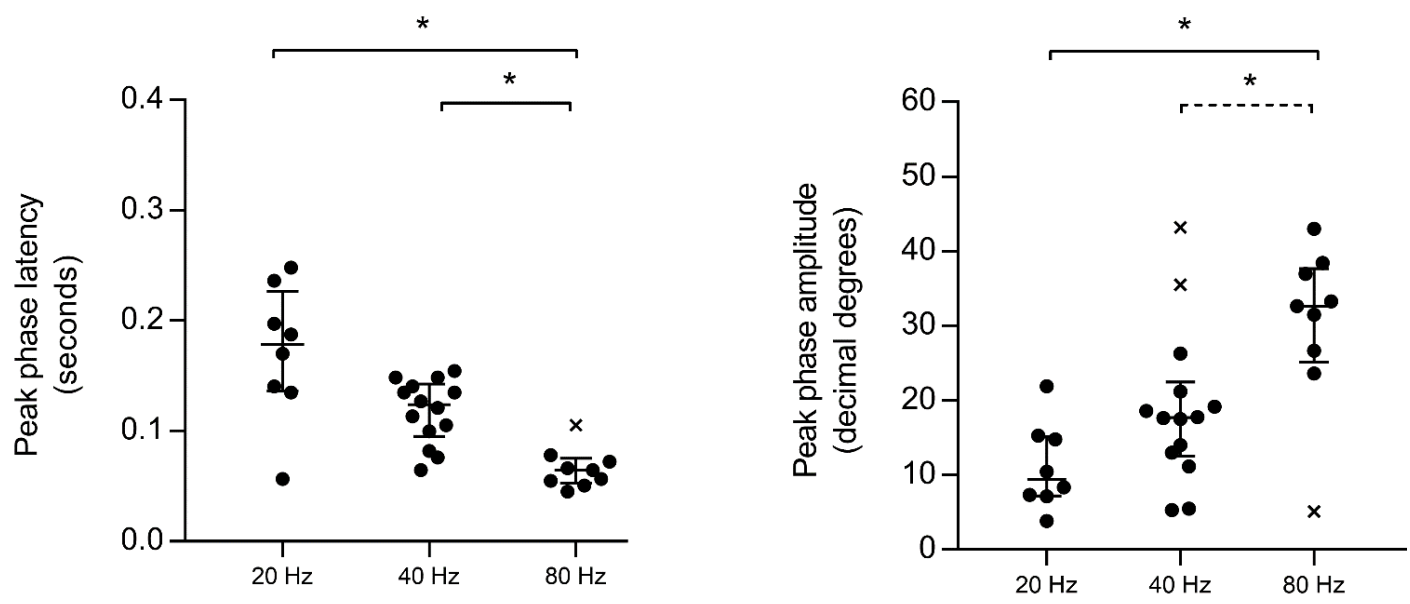

Figure 3. Overview of the peak phase latencies (left graph) and phase deviation amplitudes (right graph) per participant and per modulation condition (x-axis). Error bars indicate median values with interquartile ranges, while asterisks highlight significant differences between modulation conditions with regard to peak phase latencies or phase deviation amplitudes, respectively $(p<.017)$. The data represented in this figure are derived from IPD changes that result in transitions from $0^{\circ}$ to $180^{\circ}$. Data points represented by crosses are identified as outliers. The conditions represented by the dotted line were significantly different from each other after excluding the outliers from the analyses $(p<.017)$.

\section{CAEP Detection}

A typical example of the cortical $\mathrm{P}_{1} \mathrm{~N}_{1} \mathrm{P}_{2}$ complex is shown in Figure 4. CAEPs were detected in response to IPD changes from $0^{\circ}$ to $180^{\circ}$ in $6 / 13$ participants for the $20 \mathrm{~Hz}$ condition, in $9 / 16$ participants for the $40 \mathrm{~Hz}$ condition and in $10 / 13$ participants for the $80 \mathrm{~Hz}$ condition. A schematic overview of the latencies of $\mathrm{P}_{1}, \mathrm{~N}_{1}$, and $\mathrm{P}_{2}$ is represented in Figure 5. KruskalWallis tests $(\alpha=.05)$ with modulation frequency as independent variable and the latency (in 
seconds) of $\mathrm{P}_{1}, \mathrm{~N}_{1}$, and $\mathrm{P}_{2}$, respectively, as dependent variable did not show significant effects of modulation frequency on the latency $\left(\mathrm{P}_{1}: H(2)=2.16 ; p=.34 ; \mathrm{N}_{1}: H(2)=.28 ; p=.87 ; \mathrm{P}_{2}\right.$ : $H(2)=1.03 ; p=.60)$. For IPD changes from $180^{\circ}$ to $0^{\circ}$, the cortical $\mathrm{P}_{1} \mathrm{~N}_{1} \mathrm{P}_{2}$ complex could not be reliably detected.

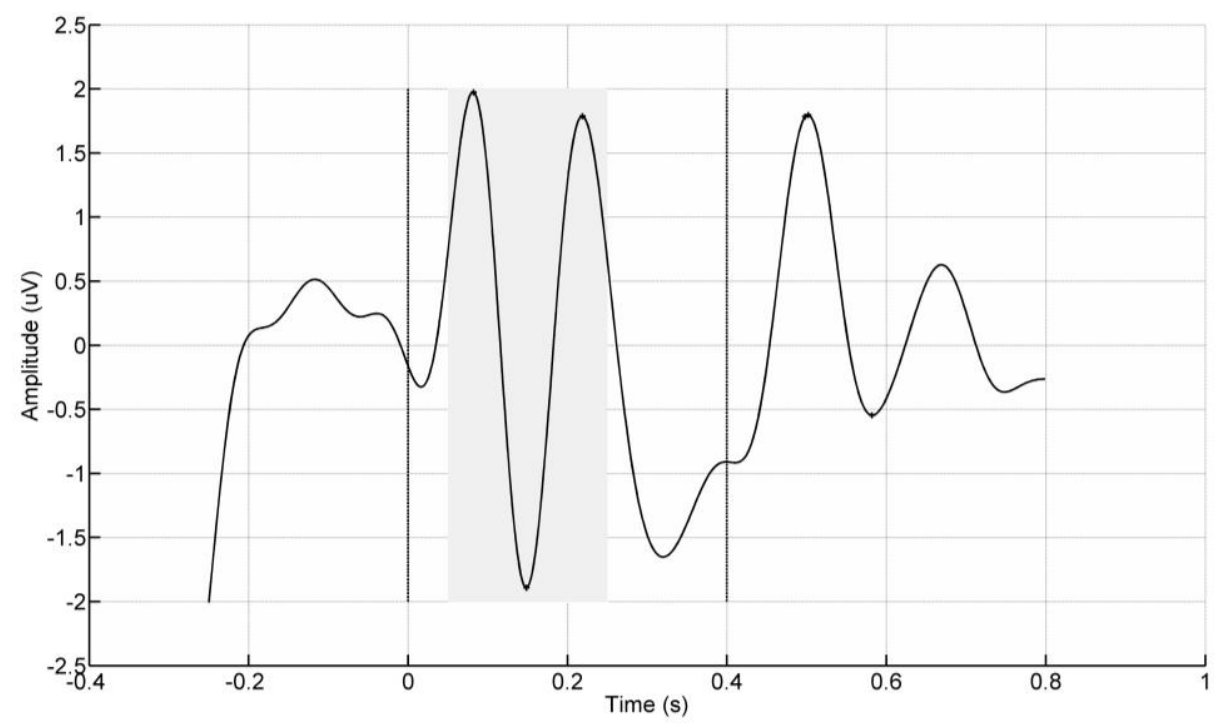

Figure 4. Typical example of the cortical $\mathrm{P}_{1} \mathrm{~N}_{1} \mathrm{P}_{2}$ complex in response to IPD changes. The EEG signal was time domain averaged over epochs ( $4 \mathrm{~s}$ in duration) and over within-stimulus IPD changes. The moment in time at which the IPD change was introduced, is referenced to 0 $\mathrm{s}$, represented by the first dashed line.

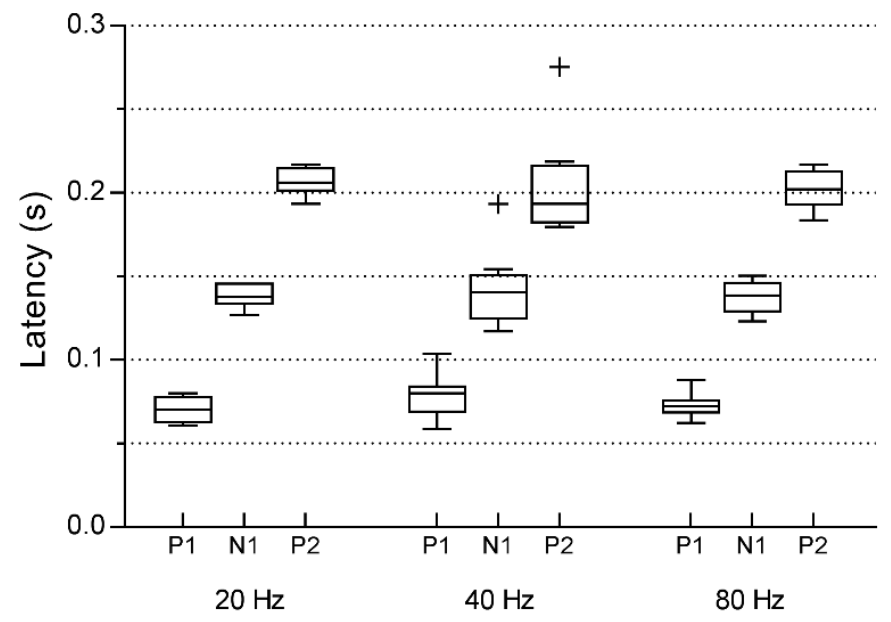

Figure 5. Tukey boxplots, representing latencies of $\mathrm{P}_{1}, \mathrm{~N}_{1}$ and $\mathrm{P}_{2}$ for the $20 \mathrm{~Hz}(\mathrm{n}=6), 40 \mathrm{~Hz}$ $(n=9)$ and $80 \mathrm{~Hz}$ conditions ( $\mathrm{n}=10)$, for IPD changes from $0^{\circ}$ to $180^{\circ}$. 


\section{Discussion}

This study investigated whether processing of IPD changes could be detected electrophysiologically as a desynchronization of ASSRs, as it was reported for a $40 \mathrm{~Hz}$ modulator in a MEG study (Ross, 2008). The possibility to extend this method to other modulation frequencies was also investigated, as well as whether this would result in different phase desynchronization patterns, based on peak phase latencies and phase deviation amplitudes.

For the $40 \mathrm{~Hz}$ condition, significant ASSR phase desynchronizations were detected in 14 out of 16 participants elicited by IPD changes from $0^{\circ}$ to $180^{\circ}$. For the reversed transitions (IPD changes from $180^{\circ}$ to $0^{\circ}$ ), a significant ASSR phase desynchronization was detected in 9 out of 16 participants. Unlike in the study by Ross (2008), there were no differences in phase deviation amplitude at group level when comparing IPD changes from $0^{\circ}$ to $180^{\circ}$ to reversed IPD changes. Ross (2008) referred to this effect as a 'response asymmetry'. A similar asymmetry was detected in the work of Magezi and Krumbholz (2010). They measured evoked responses to changes in interaural time differences (ITDs) using noise stimuli. Changes in ITDs from zero to a lateralized percept resulted in larger responses than the other way around, which could favor an opponent channel model over the topographic model for ITD processing in humans. In our study, the asymmetry might be reflected at group level by the Hotelling $T^{2}$ test reaching significance in more participants for IPD changes from $0^{\circ}$ to $180^{\circ}$ than for the reversed changes. It must be noted, however, that the size of the phase deviation amplitudes for a $40 \mathrm{~Hz}$ modulator were overall lower in our study (median value of $18^{\circ}$ ) than expected based on the work by Ross (2008) (mean value of $50^{\circ}$ ). This could potentially be explained by methodological differences. As steady-state responses can result from multiple sources (Picton et al, 2003) and therefore, might contain different temporal dynamics of phase change, results between MEG and EEG studies might not completely correspond, especially as electrical and 
neuromagnetic measurements are presumably more responsive to different sources throughout the auditory pathway (Herdman et al, 2002; Ross et al, 2003). For the $40 \mathrm{~Hz}$ condition, however, the median points in time at which the phase deviations reached their maximum in our study ( 0.12 to $0.14 \mathrm{~s})$ were comparable to the grand average time lag of $0.14 \mathrm{~s}$, as reported by Ross (2008).

For the $20 \mathrm{~Hz}$ and $80 \mathrm{~Hz}$ conditions, respectively, significant desynchronizations of ASSR phase were detected in response to IPD changes from $0^{\circ}$ to $180^{\circ}$ in 8 and 9 out of 13 participants. Similar to the $40 \mathrm{~Hz}$ condition, significant ASSR phase desynchronizations were present in less participants for the reversed IPD changes (from $180^{\circ}$ to $0^{\circ}$ ): significant phase desynchronizations were detected in 4 out of 13 participants for $20 \mathrm{~Hz}$ and in 6 out of 13 participants for $80 \mathrm{~Hz}$. Based on these results, it can be concluded that phase locked steadystate neural activity desynchronizes in response to IPD changes and that this happens independent of the three modulation frequencies applied in this study, even though measurement times were up to twice as long for the 20 and $80 \mathrm{~Hz}$ conditions. For all three modulation conditions, IPD changes from $0^{\circ}$ to $180^{\circ}$ resulted in more robust results than IPD changes from $180^{\circ}$ to $0^{\circ}$.

As the lateral and medial superior olives are the first stage of the central auditory system at which binaural information is exchanged between left and right auditory pathways (e.g. Tollin \& Yin, 2005; Grothe et al, 2010), it is expected that it would be possible to measure binaural functioning based on electrophysiological measures of brainstem activity. By investigating $80 \mathrm{~Hz}$ ASSRs, which are predominantly generated by brainstem generators (Giraud et al, 2000; Herdman et al, 2002), and by providing evidence for these ASSRs to desynchronize in response to IPD changes, our study could support the limited evidence available in literature for electrophysiological correlates of binaural interaction at brainstem level (Wilson \& Krishnan, 2005; Clinard et al, 2016). To investigate whether the underlying 
physiological processes of the phase deviations in the 20,40 , and $80 \mathrm{~Hz}$ conditions indeed differed from each other, the morphology of the phase deviations over the three modulation conditions were compared. For IPD changes from $0^{\circ}$ to $180^{\circ}$, our data showed that modulation frequency significantly affected both peak phase latency and phase deviation amplitude. The point in time at which the phase deviations reached their maximum was detected significantly earlier for $80 \mathrm{~Hz}$ than for 20 and $40 \mathrm{~Hz}$. The size of the phase deviation amplitudes was also significantly larger for $80 \mathrm{~Hz}$ compared to $20 \mathrm{~Hz}$. The latencies of the CAEPs (see Figure 6), however, were similar for all three stimulus conditions and comparable to those reported by Tremblay et al (2007) and by Ross et al (2007b) ( $\left.\mathrm{P}_{1}: 65 \mathrm{~ms}, \mathrm{~N}_{1}: 134 \mathrm{~ms}, \mathrm{P}_{2}: 226 \mathrm{~ms}\right)$.

The ASSR results suggest that desynchronization of phase locked steady state activity in response to IPD changes behaves differently when the activity is recorded from predominantly brainstem generators $(80 \mathrm{~Hz}$ condition) than from increasing cortico-thalamic neural generators (40 and $20 \mathrm{~Hz}$ conditions). One could, however, also explain the faster restoration of the $80 \mathrm{~Hz}$ phase deviations compared to those of lower modulation frequencies by its faster oscillation rate, which yields more cycles for neural activity to phase lock to. Unfortunately, not a lot of data is available on the time course of $80 \mathrm{~Hz}$ ASSRs compared to the time course of $40 \mathrm{~Hz}$ ASSRs (e.g. Ross et al, 2002; Ross et al, 2005; Ross et al, 2007a; Ross, 2008). Future wise, it would be interesting to take a closer look at the $80 \mathrm{~Hz}$ ASSR phase to determine if it behaves similarly when it synchronizes after stimulus onset as after IPD related desynchronization, such as is reported for $40 \mathrm{~Hz}$ ASSRs (Ross et al, 2002; Ross, 2008).

Overall, the method described in this study could be a valuable tool to objectively assess how we process changes in binaural information. In particular, its applicability at an individual level shows potential for both clinical and research purposes. Future research, however, is necessary to look into reducing testing time, simplifying the 64 channel set-up and investigating the variability of the responses, in close correspondence to a behavioral task. Nevertheless, the 
results are promising and a step forward to an objective measure that could be used, for instance, to investigate binaural cue processing across the lifespan and/or as a tool to optimize hearing aid fitting strategies with respect to binaural hearing.

\section{Conclusions}

ASSR desynchronization to IPD changes was successfully recorded in this study using EEG. It proved feasible for 20,40 , and $80 \mathrm{~Hz}$ modulators and for all modulation conditions, IPD changes from $0^{\circ}$ to $180^{\circ}$ resulted in more robust results than IPD changes from $180^{\circ}$ to $0^{\circ}$. Furthermore, desynchronization and restoration of ASSR phase took place significantly faster for $80 \mathrm{~Hz}$ than for $40 \mathrm{~Hz}$ and $20 \mathrm{~Hz}$. The latencies of the CAEPs were similar for all three modulation conditions, as expected.

The results presented in this study show the potential of using this method for both clinical and research purposes. Therefore, this study contributes to developing tools to objectively asses how we process changes in binaural information and to understand the underlying neural mechanisms.

\section{Acknowledgements}

We want to thank all participants who made time to participate in this study. The project was financed by the Research Council of KU Leuven (grant support: OT/12/98) and the European Research Council (ERC) under the European Union's Horizon 2020 research and innovation programme (grant agreement No 637424, ERC starting Grant).

Portions of the data presented in this technical report were presented by Charlotte Vercammen at HEaring Across the Lifespan at Lake Como, Italy (June 5-7, 2014) and at the 
Twelfth Congress of The European Federation of Audiology Societies (EFAS) at the Green Park Pendik Convention Centre, Istanbul, Turkey (May 27-30, 2015).

The authors report no conflict of interest. 


\section{References}

Aickin, M. \& Gensler, H. 1996. Adjusting for multiple testing when reporting research results: The Bonferroni vs Holm methods. Am J Public Health, 86, 726-728.

American Clinical Neurophysiology Society. 2006. Guideline 5: Guidelines for Standard Electrode Position Nomenclature. Am J Electroneurodiagnostic Technol, 46, 222-225.

Biosemi. 2010. Amsterdam: Biosemi B.V.

Carhart, R. \& Jerger, J. 1959. Preferred method for clinical determination of pure-tone thresholds. J Speech Hear Disord, 24, 330-345.

Cebulla, M., Stürzebecher, E. \& Elberling, C. 2006. Objective detection of auditory steadystate responses: comparison of one-sample and q-sample tests. J Am Acad Audiol, 17(2), 93-103.

Clinard, C. G., Hodgson, S. L. \& Scherer, M. E. 2016. Neural Correlates of the Binaural Masking Level Difference in Human Frequency-Following Responses. J Assoc Res Otolaryngol, advance online publication. doi: 10.1007/s10162-016-0603-7

Dajani, H.R. \& Picton, T.W. 2006. Human auditory steady-state responses to changes in interaural correlation. Hear Res, 219, 85-100.

Debruyne, F. 1984. Binaural interaction in early, middle and late auditory evoked responses. Scand Audiol, 13, 293-296.

de Cheveigné, A. \& Simon, J.Z. 2008. Denoising based on spatial filtering. J Neurosci Methods, 171, 331-339.

Dobie, R. A. \& Berlin, C.I. 1979. Binaural interaction in brainstem-evoked responses. Arch Otolaryngol, 105, 391-398.

Dobie, R. A. \& Norton, S. J. 1980. Binaural interaction in human auditory evoked potentials. Electroencephalogr Clin Neurophysiol, 49, 303-313.

Dobie, R. A. \& Wilson, M. J. 1994. Objective detection of 40-Hz auditory evoked potentials: phase coherence vs. magnitude-squared coherence. Electroencephalogr Clin Neurophysiol, 92, 405-413.

Galambos, R. \& Makeig, S. 1992. Physiological studies of central masking in man. I: The effects of noise on the 40-Hz steady-state response. J Acoust Soc Am, 92, 2683-2690.

Giraud, A.-L., Lorenzi, C., Ashburner, J., Wable, J., Johnsrude, I., et al. 2000. Representation of the Temporal Envelope of Sounds in the Human Brain. J Neurophysiol, 84, 1588-1598.

Grothe, B., Pecka, M. \& Mcalpine, D. 2010. Mechanisms of Sound Localization in Mammals. Physiol Rev, 90, 983-1012.

Haywood, N. R., Undurraga, J. A., Marquardt, T. \& Mcalpine, D. 2015. A Comparison of Two Objective Measures of Binaural Processing. Trends Hear, 19, 1-17.

Herdman, A. T., Lins, O., Van Roon, P., Stapells, D. R., Scherg, M., et al. 2002. Intracerebral sources of human auditory steady-state responses. Brain Topogr, 15, 69-86.

Hofmann, M. \& Wouters, J. 2010. Electrically Evoked Auditory Steady State Responses in Cochlear Implant Users. J Assoc Res Otolaryngol, 11, 267-282.

Hotelling, H. 1931. The generalization of student's ratio. Ann Math Stat, 2, 360-378. 
IBM Corp. 2013. SPSS Statistics for Windows (version 23.0) [Computer program]. Armonk, NY.

Iglewicz, B. \& Banerjee, S. 2001. A simple univariate outlier identification procedure. Proc Annu Meet Am Stat Assoc, Statistical Computing Section. CD-ROM.

Kevanishvili, Z. \& Lagidze, Z. 1987. Masking level difference: an electrophysiological approach. Scand Audiol, 16, 3-11.

Kimber, A. C. 1990. Exploratory Data Analysis for Possibly Censored Data from Skewed Distributions. J R Stat Soc Ser C (Applied Stat), 39, 21-30.

Klumpp, R. G. \& Eady, H. R. 1956. Some Measurements of Interaural Time Difference Thresholds. J Acoust Soc Am, 28, 859-860.

Magezi, D.A. \& Krumbholz, K. 2010. Evidence for opponent-channel coding of interaural time differences in human auditory cortex. J Neurophysiol, 104, 1997-2007.

The MathWorks Inc. 2013. Matlab (version R2103a) [Computer program]. Natick, MA.

McPherson, D. L. \& Starr, A. 1993. Binaural interaction in auditory evoked potentials: Brainstem, middle- and long-latency components. Hear Res, 66, 91-98.

Nakagawa, S. 2004. A farewell to Bonferroni: The problems of low statistical power and publication bias. Behav Ecol, 15, 1044-1045.

Picton, T. W., John, M. S., Dimitrijevic, A. \& Purcell., D. 2003. Human auditory steady-state responses. Int J Audiol, 42(4), 177-219.

Rayleigh, F. R. S. 1880. XII. On the resultant of a large number of vibrations of the same pitch and of arbitrary phase. Philos Mag J Sci fifth Ser, 10, 73-78.

Ross, B. 2008. A novel type of auditory responses: temporal dynamics of 40-Hz steady-state responses induced by changes in sound localization. J Neurophysiol, 100, 1265-1277.

Ross, B., Draganova, R., Picton, T. W. \& Pantev, C. 2003. Frequency specificity of 40-Hz auditory steady-state responses. Hear Res, 186, 57-68.

Ross, B., Fujioka, T., Tremblay, K. L. \& Picton, T. W. 2007a. Aging in binaural hearing begins in mid-life: evidence from cortical auditory-evoked responses to changes in interaural phase. J Neurosci, 27, 11172-11178.

Ross, B., Herdman, A. T. \& Pantev, C. 2005. Stimulus induced desynchronization of human auditory 40-Hz steady-state responses. J Neurophysiol, 94, 4082-4093.

Ross, B., Picton, T. W. \& Pantev, C. 2002. Temporal integration in the human auditory cortex as represented by the development of the steady-state magnetic field. Hear Res, 165, 6884.

Ross, B., Tremblay, K. L. \& Picton, T. W. 2007b. Physiological detection of interaural phase differences. J Acoust Soc Am, 121, 1017-1027.

Tollin, D. J. \& Yin, T. C. T. 2005. Interaural Phase and Level Difference Sensitivity in LowFrequency Neurons in the Lateral Superior Olive. J Neurosci, 25, 10648-10657.

Tremblay, K., Picton, T. W. \& Ross, B. 2007. Auditory evoked MEG responses to interaural phase changes: Effects of aging on response latencies. Int Congr Ser, 1300, 69-72.

Wilson, J. R. \& Krishnan, A. 2005. Human frequency-following responses to binaural masking 
level difference stimuli. J Am Acad Audiol, 16, 184-195.

Wong, W. Y. S. \& Stapells, D. R. 2004. Brain stem and cortical mechanisms underlying the binaural masking level difference in humans: an auditory steady-state response study. Ear Hear, 25, 57-67.

Yost, W. A. \& Dye, R. H. 1988. Discrimination of interaural differences of level as a function of frequency. J Acoust Soc Am, 83, 1846-1851.

Zhou, J. \& Durrant, J. D. 2003. Effects of interaural frequency difference on binaural fusion evidenced by electrophysiological versus psychoacoustical measures. J Acoust Soc Am, $114,1508-1515$.

Zwislocki, J. \& Feldman, R. S. 1956. Just noticeable differences in dichotic phase. J Acoust Soc Am, 28, 860-864. 